\title{
High resolution crystallographic and chemical characterisation of iodine induced stress corrosion crack tips formed in irradiated and non-irradiated zirconium alloys \\ DOI:
}

10.1016/j.jnucmat.2019.03.027

\section{Document Version}

Accepted author manuscript

Link to publication record in Manchester Research Explorer

\section{Citation for published version (APA):}

Gillen, C., Garner, A., Jones, C., Moore, K., Tejland, P., \& Frankel, P. (2019). High resolution crystallographic and chemical characterisation of iodine induced stress corrosion crack tips formed in irradiated and non-irradiated zirconium alloys. Journal of Nuclear Materials, 519, 166-172. https://doi.org/10.1016/j.jnucmat.2019.03.027

\section{Published in:}

Journal of Nuclear Materials

\section{Citing this paper}

Please note that where the full-text provided on Manchester Research Explorer is the Author Accepted Manuscript or Proof version this may differ from the final Published version. If citing, it is advised that you check and use the publisher's definitive version.

\section{General rights}

Copyright and moral rights for the publications made accessible in the Research Explorer are retained by the authors and/or other copyright owners and it is a condition of accessing publications that users recognise and abide by the legal requirements associated with these rights.

\section{Takedown policy}

If you believe that this document breaches copyright please refer to the University of Manchester's Takedown Procedures [http://man.ac.uk/04Y6Bo] or contact uml.scholarlycommunications@manchester.ac.uk providing relevant details, so we can investigate your claim.

\section{OPEN ACCESS}




\title{
High resolution crystallographic and chemical characterisation of iodine induced stress corrosion crack tips formed in irradiated and non-irradiated zirconium alloys
}

\author{
Conor Gillen ${ }^{1 *}$, Alistair Garner ${ }^{1}$, Pia Tejland², Philipp Frankel ${ }^{1}$
}

'Materials Performance Centre, School of Materials, The University of Manchester, Manchester M13 9PL, UK

2Studsvik Nuclear AB, SE-611 82 Nyköping, Sweden

${ }^{*}$ Corresponding Author

\begin{abstract}
High resolution crystallographic and chemical analysis has been performed on iodine-induced stress corrosion crack tips taken from irradiated and non-irradiated zirconium alloys. Defuelled cladding tubes were subjected to mandrel testing and subsequent analysis of the crack tip region showed the predominance of transgranular cracking on non-basal planes for the first time in irradiated material. In a separate mandrel-tested crack tip sample, iodine was unambiguously identified in the crack tip region using both STEM-EDX and NanoSIMS. For comparison, non-irradiated C-ring samples were subjected to testing in iodine-ethanol solution, subsequent crack tip analysis showed large levels of intergranular attack and no significant iodine segregation.
\end{abstract}

\section{Introduction}

Pellet cladding interaction $(\mathrm{PCl})$ is a fuel failure phenomenon in light water nuclear reactors that occurs due to thermal expansion of the fuel pellet and fission gas swelling, resulting in tensile hoop stresses at regions where the fuel contacts the inner surface of the cladding. In the presence of corrosive fission products, such as iodine, this can lead to iodine-induced stress corrosion cracking (I-SCC). I-SCC was first identified in the 1960s, with a large research effort culminating in preventative measures such as careful control of power ramps and $\mathrm{PCl}$ resistant cladding liners. This dramatically reduced the number of $\mathrm{PCl}$ failures, with bundle defect rates of $<0.01 \%$ in Canada from 2000 - 2005 [1]. However with the increased use of renewable energy, there is a drive to better understand the mechanisms of $\mathrm{PCl}$ so that over conservative operational restrictions can be lifted. This is especially 
important during extended reduced power operation (ERPO), after which the risk of $\mathrm{PCl}$ failure is significantly increased [2].

As a result of the mitigation measures, research into mechanistic understanding of $\mathrm{PCl}$ has decreased over the past decades. Therefore, many modern characterisation methods that have been successful in advancing understanding other types of SCC, have not yet been applied to $\mathrm{PCl}$. In steels, used widely as structural materials in nuclear reactors, multiple analytical techniques have been applied to the same stress corrosion crack tip to extract chemical and microstructural information at multiple length scales [3]. 3D techniques (Xray diffraction and electron microscopy) and advanced high resolution analytical techniques (electron backscatter diffraction and atom probe tomography) have led to greater understanding of crack propagation mechanisms and to the design of more SCC resistant alloys [4-6].

In comparison to more widely studied SCC phenomenon, significant gaps remain in the understanding of the specific mechanisms that control $\mathrm{PCl}$ crack initiation and propagation in $\mathrm{Zr}$ alloys, in particular the role of microchemistry at the crack tip. Cracks are thought to progress initially intergranularly, and then mainly transgranularly; depending upon the magnitude of the stress and the orientation of local grains [7-9]. Transgranular crack propagation during I-SCC is frequently reported to occur on exclusively basal planes [8,1012]. The presence of iodine is postulated to weaken these planes, resulting in cleavage at significantly lower stresses than would be expected without iodine, although the exact mechanism is unclear. Cracks pass directly through favourably orientated grains, cleavage planes in neighbouring grains are then connected through prismatic fluting should they not be aligned, with both processes together termed pseudo-cleavage. By varying texture and measuring crack susceptibility $[10,13]$, or by using $x$-ray diffraction [11], basal cleavage has been identified as the responsible transgranular cracking mechanism in unirradiated material. However, the effect of grain orientation on crack propagation in irradiated material is less clear, there is limited evidence that the effect of texture is reduced in irradiated material [14] and it has been shown that many metallurgical factors that affect I-SCC susceptibility in non-irradiated material become negligible above a critical irradiation dose [8] [Cox review]. Proton and neutron irradiation have been observed to change the hierarchy of slip system activation via dislocation channelling [15] which could affect the texture dependence and contribute to increased susceptibility of zirconium alloys to I-SCC after irradiation [16].

Due to the expense and difficulties associated with irradiated materials testing, laboratory tests are widely used to increase the testing matrix. Often these tests are 
performed are in alcoholic iodine solutions on non-irradiated material and so it is important to evaluate the validity of these tests in the context of in-reactor stress corrosion cracking. This study aims to give new insight into the mechanisms of in-reactor I-SCC propagation in $\mathrm{Zr}$ alloys using modern analytical techniques on realistically simulated $\mathrm{PCl}$ cracks in neutronirradiated material and through comparison with a non-irradiated crack tip from a laboratory test. In-reactor stress corrosion cracking is emulated through a mandrel test, which uses a ceramic insert to impart a realistic stress state on the inner surface of defueled cladding from a reactor in an iodine vapour environment. In contrast, non-irradiated samples have been tested by compression of a C-ring sample whilst immersed in iodine-ethanol solution. In both cases, thin foils from cracks tips are prepared using a focused ion beam (FIB) and analysed using transmission Kikuchi diffraction in the scanning electron microscope (TKDSEM) and electron dispersive spectroscopy using scanning transmission electron microscopy (STEM-EDX).

\section{Materials}

For the mandrel tests the material used was non-liner recrystallised Zircaloy-2 supplied by Framatome $\mathrm{GmbH}$. The material was produced according to the Low Temperature Process (LTP), which is standard for BWR claddings and leads to an equiaxed microstructure (with grain size $\sim 2-3 \mu \mathrm{m}$ ) and a pronounced radial crystallographic texture. The cladding had previously been irradiated in a Swedish boiling water reactor (BWR) to a burn up of 21.2 $\mathrm{MWd} / \mathrm{kg}$. The mandrel test was conducted at Studsvik, the sample was tested at a strain rate of $21.6 \% / \mathrm{hr}$ at an iodine partial pressure of $100 \mathrm{pA}$. A schematic is shown in Figure 1 (a), more details of the experimental set-up can be found in [17]. For the C-ring compression tests the material used was recrystallised ZIRLO' supplied by Westinghouse, manufactured using standard routes for cladding tubes, leading to an average grain size of $\sim 2 \mu \mathrm{m}$ and a strong radial texture. The C-rings were machined directly from the cladding tube and tested at a constant load in iodine-ethanol solution of concentration of $1 \mathrm{~g} / \mathrm{L}$. A schematic is shown in Figure 1 (b) and more details of the set up can be found in $[9,18]$ ZIRLO was originally chosen for the C-ring tests due to renewed interest in $\mathrm{PCl}$ behaviour of PWR cladding alloys with the increased use of extended low power operation. Irradiated Zircaloy-2 material was the only material available from the mandrel tests, highlighting the difficulties with availability of irradiated material. It should therefore be highlighted that two different alloys were used, only one of which (Zircaloy-2) has been irradiated. The chemical compositions of each alloy are shown in Table $1[19,20]$. Both alloys have similar textures 
and grain size however due to the different alloy compositions, most notably the addition of $\mathrm{Nb}$ and reduction of $\mathrm{Sn}$ in ZIRLO, there are differences in mechanical properties between the alloys that could affect the results presented here. This will be discussed in more detail later.

Table 1: Composition in wt.\% of ZIRLO and Zircaloy-2 [20] alloys used in this study

\begin{tabular}{ccccccc}
\hline Element & Cr & Fe & Nb & Ni & Sn & Zr \\
\hline ZIRLO (wt.\%) & $<0.01$ & 0.09 & 0.87 & $<0.01$ & 0.92 & bal. \\
Zircaloy-2 (wt.\%) & 0.1 & 0.15 & -- & 0.05 & 1.5 & bal. \\
\hline \hline
\end{tabular}

(a)

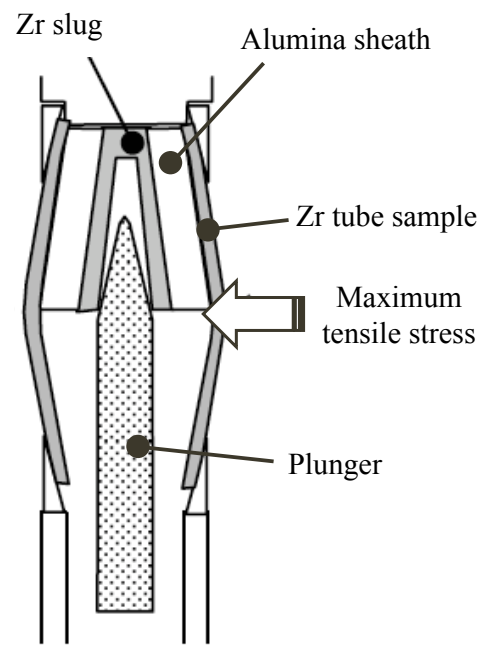

(b)

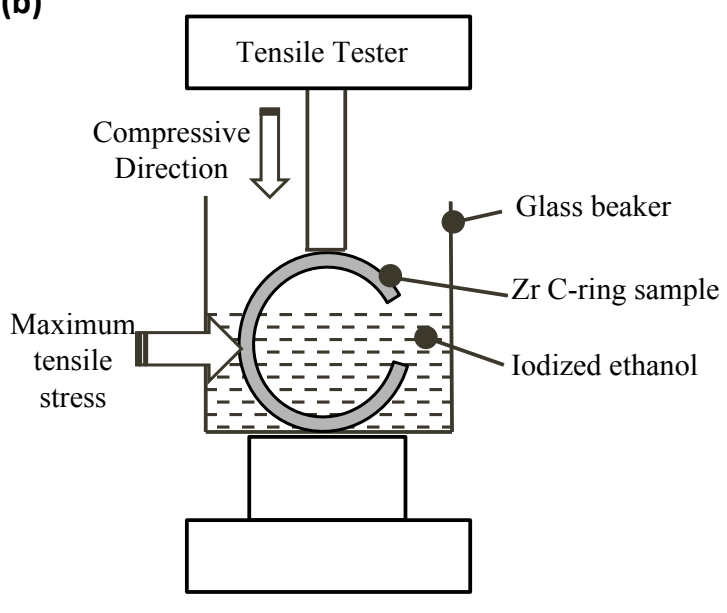

Figure 1: Schematics of experimental setups for (a) mandrel testing of tube samples and (b) compression testing of $C$-ring samples

\section{Experimental}

\subsection{FIB sample preparation}

FIB liftouts from the mandrel-tested irradiated material were performed using a Zeiss Crossbeam FIB-SEM situated in the hot laboratory at Studsvik specifically for radioactive sample preparation. Due to geometric constraints, a unique procedure for lifting out crack tips was developed which allows for samples to be lifted out perpendicular to the sample surface, an outline of this procedure is shown in Figure 2. An incipient was firstly identified and the ring section ground from the radial direction until the crack tips just become visible, as shown in Figure 2(a). Trenches are then dug around the crack tips in order to identify 
potential crack tip regions below the surface, once one is identified, the region is protected using Pt and trenches are dug either side to isolate the crack tip region. The sample is then lifted out and attached to a $\mathrm{Cu}$ grid using the in-situ liftout procedure and thinned to electron transparency using standard methods. The main benefit of this method is that it allows for the liftout of a suitable sample perpendicular to the sample surface, which was necessary to geometrical constraints in the active FIB. For the C-ring samples, FIB liftouts were prepared using the standard in-plane procedure for crack tips according to [21] using an FEI Helios 660 FIB-SEM. A reduced overtilt during the low KeV cleaning stage was used in order to reduce FIB-induced hydride artefacts [22].
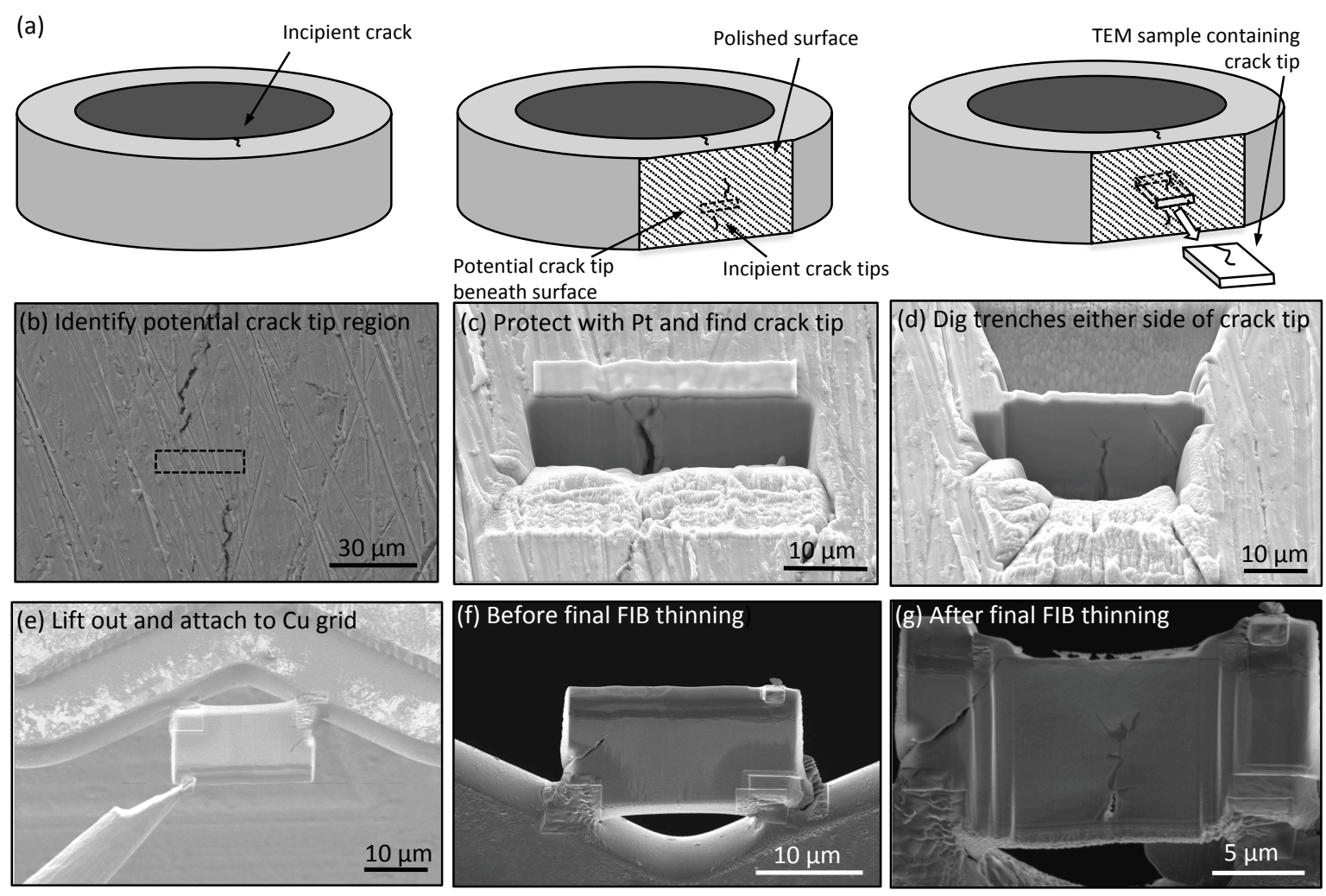

Figure 2: (a) Schematic of TEM sample preparation route for irradiated tested samples, (b) - (g) FIB-SEM images of TEM sample preparation route

\subsection{TKD}

TKD was performed using an FEI Magellan FEG SEM operated at $30 \mathrm{kV}$ with a current of 1.6nA. The FIB sample was mounted in transmission geometry tilted at $160^{\circ}$, more details can be found in [23]. The patterns were collected at $\sim 20 \mathrm{~Hz}$ using $4 \times 4$ binning and indexed using the AZTEC software package developed by Oxford Instruments HKL. 


\subsection{STEM-EDX}

Energy dispersive X-ray spectroscopy (EDS) chemical mapping was performed using an FEl Talos F200X microscope operated at $200 \mathrm{keV}$ with a probe current of $180 \mathrm{pA}$ and fitted with the FEI ChemiSTEM ${ }^{\text {TM }}$ system. The chemical maps are shown in individually scaled net counts from background subtracted spectra using FEI Velox software.

\subsection{NanoSIMS}

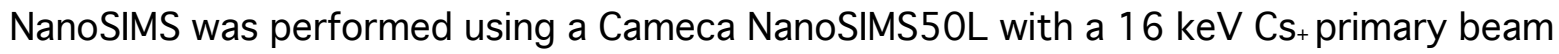
and a beam current of 0.86-1.64 pA. The detectors were set to simultaneously detect secondary electrons (SE), ${ }^{90} \mathrm{Zr},{ }^{16} \mathrm{O}^{-}$and ${ }^{127} \mathrm{I}^{-}\left({ }^{12} \mathrm{C}-,{ }^{56} \mathrm{Fe}^{16} \mathrm{O}^{-}\right.$and ${ }^{90} \mathrm{Zr}^{16} \mathrm{O}^{-}$signals were also collected but not shown here). The beam was rastered over a $10 \times 10 \mu \mathrm{m}$ region surrounding the crack tip with a dwell time of $4 \mathrm{~ms}$. A total of 160 images were collected from the same crack tip region without prior implantation in order to minimise material removal on the thin foil. ImageJ with the OpenMIMS plugin (Harvard, Cambridge, MA, USA) was used to analyse the images. 

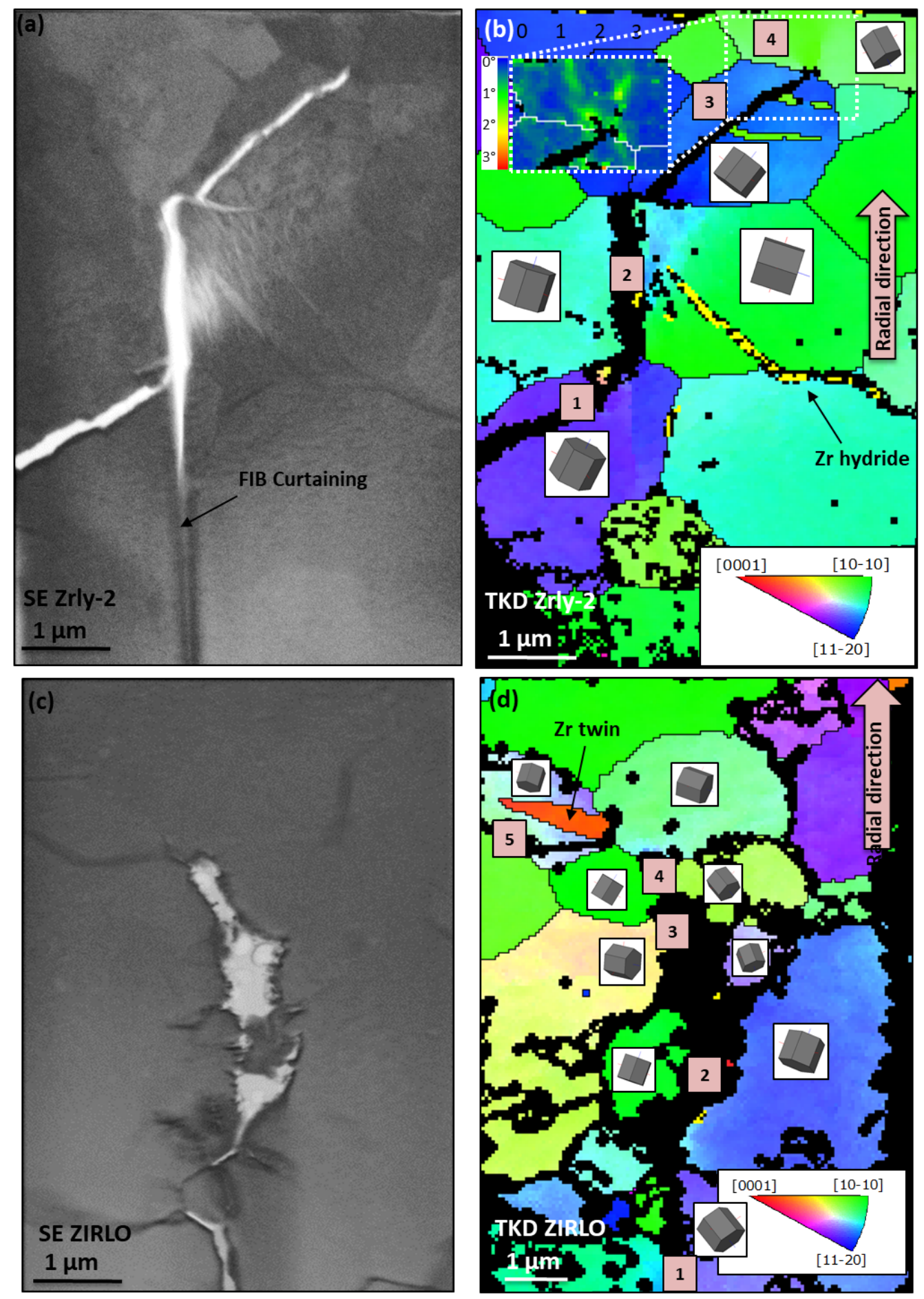

Figure 3: Secondary electron images and TKD orientation maps in IPF // axial direction colouring for crack tip regions from (a), (b) tested irradiated Zircaloy-2 and (c), (d) C-ring tested ZIRLO. (b) inset shows local misorientation of outlined crack tip region. The original radial direction of the cladding tube is marked on each map, the maximum tensile stress is assumed to be approximately perpendicular to the radial direction in both experiments. 


\section{Results and Discussion}

The TKD orientation map, and corresponding secondary electron (SE) image, of a crack tip region formed during mandrel testing is shown in Figure 3 (a) and (b). The SE image shows a planar crack path that appears to be changing direction on the order of the grain size of the material $(2-3 \mu \mathrm{m})$. It is worth pointing out that the sizes and orientations of the grains in the regions of interest are comparable in the two materials. The contrast within the grains results from fine scale FIB-induced hydride artefacts [22], however they are not considered to affect the orientation of the matrix grains and so are excluded from this analysis. The orientation map of this region reveals four distinct cracking regions, which have been labelled in Figure 3 (b). The orientations are also represented by the HCP unit cell of each relevant grain in order to aid visualisation of the local crystallography. Region 1 shows clear transgranular (TG) failure on a pyramidal plane ( $60^{\circ}$ from basal). The crack then continues into region 2, where an intergranular cracking mechanism is observed along a high angle grain boundary (GB) with a misorientation angle-axis of $90^{\circ}$ [1 $\left.\overline{2} 10\right]$. A large hydride platelet is also observed in one of these grains (annotated on Figure $3(b)$ ), due to the size and morphology of this hydride it is thought that this was a pre-existing hydride and not the result of FIB preparation, However there is no interaction between the hydride and the intergranular crack In region 3 , the crack reverts to a transgranular path along a prismatic plane $\left(\sim 80^{\circ}\right.$ from basal) until it reaches the grain in region 4 . This grain is oriented to allow for basal cleavage, however the crack terminates here. It should be noted that the crack could have continued out of the plane imaged, however the crack has not continued transgranularly though this grain despite local misorientation indicating local strain as shown in the inset of Figure 3(b). The high levels of local misorientation in this region demonstrate clearly that there were stresses that were sufficient to cause deformation close to this grain boundary, inducing significant deformation but not causing fracture.

For comparison, the TKD orientation map and SE image from the crack tip region of a non-irradiated C-ring are shown in Figure 3 (c) and (d). The SE image shows a less planar, complex crack morphology in this sample, characteristic of intergranular corrosion/cracking in zirconium alloys [24]. In addition, significant degradation of the GBs surrounding the crack tip can be observed in the SE image. The TKD orientation map shows a clear preference for intergranular cracking in this crack tip region. The four regions all show intergranular cracking along high angle GBs characterised by the following misorientation angle/axes respectively:

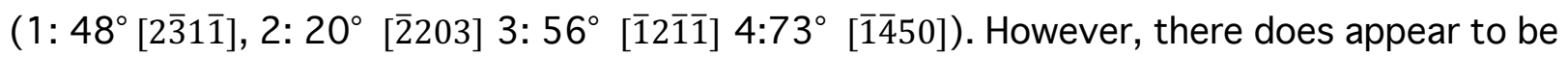
one region of near-basal transgranular cracking (labelled as region 5 in the figure). The crack through this grain is approximately parallel to the loading axis, so there should be minimal 
applied stress normal to the crack. It is therefore possible that crack propagation is influenced by residual stress in this region. Although transgranular cracking is known to occur in samples tested in alcoholic solutions, a tendency for increased levels of IG in comparison to samples tested in gaseous iodine has been previously reported $[8,25,26]$. HAADF STEM images and corresponding STEM-EDX chemical maps of the same region are shown in Figure 4 (e) - (h). The HAADF images show the complex crack tip morphology and extensive grain boundary degradation as shown in the SE image. The Zr chemical map shows that removal of significant levels of $\mathrm{Zr}$ at GBs surrounding the crack tip. Interestingly, it appears not all of the GBs surrounding the crack tip are attacked, amongst these protective boundaries are GBs characterised by the following misorientation angle-axes: $78^{\circ}[\overline{3} \overline{4} 70]$ and $48^{\circ}$ [3251]. The oxygen map shows significant oxidation in the attacked GBs, indicating that the inner surfaces were exposed to air after the test. The iodine map shows no iodine segregation to these attacked GBs or ahead of the crack tip.
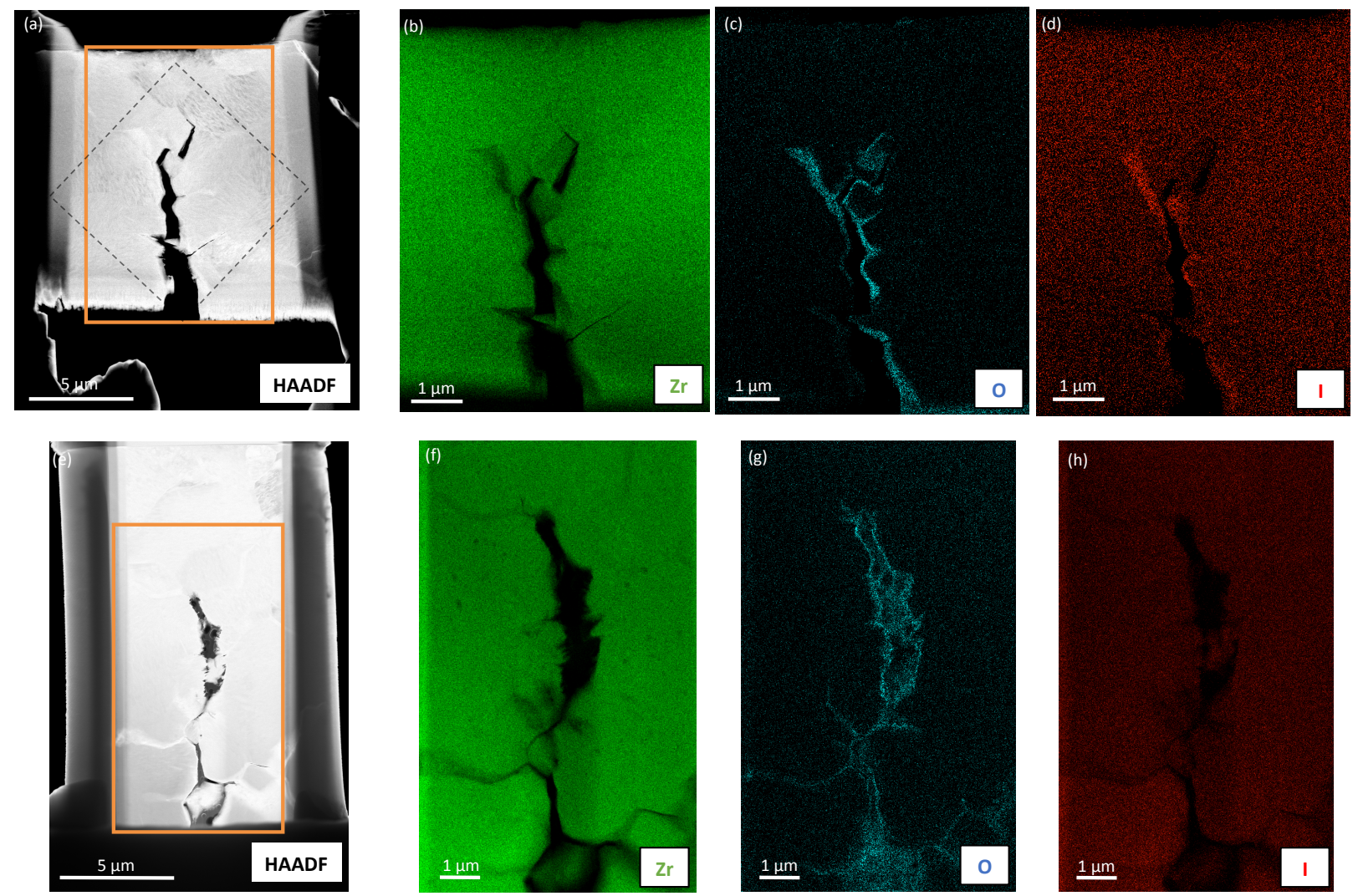

Figure 4: HAADF-STEM images showing crack tip regions from (a) a second crack from mandrel tested irradiated Zircaloy-2 in gaseous iodine and (e) the ZIRLO compression tested in iodized ethanol. Corresponding STEM-EDX chemical maps from orange highlighted regions are shown in $(b)-(d)$ and $(f)$-( $h)$ respectively. Region for subsequent NanoSIMS analysis is highlighted by dashed lines in (a). 
The HAADF STEM image and corresponding STEM-EDX chemical maps of a separate crack tip lifted out from the same irradiated mandrel-tested sample are shown in Figure 4 (a) (d). The HAADF image again shows a more planar crack morphology than in the crack tip tested in iodine ethanol solution. The $\mathrm{Zr}$ chemical map shows limited amounts of GB degradation around the crack tip, however, there appears to be a thick band of reduced $\mathrm{Zr}$ intensity ahead of the open crack tip. This can be seen to be heavily oxidised in the oxygen chemical map (Figure 4 (c)), which is most likely to have occurred post-test. The iodine chemical map suggests that this thick band is also enriched with iodine. Due to difficulties with separating iodine and oxygen signals using EDX caused by overlapping of the characteristic X-ray peaks, an alternative technique was required to confirm the presence of iodine at the crack tip. A NanoSIMS acquisition was therefore performed in the area highlighted in Figure 4 (a), and resultant NanoSIMS chemical maps are shown in Figure 5. The distribution of chemical species is in good agreement with the STEM-EDX results, unambiguously confirming the presence of iodine at the crack tip in this irradiated mandreltested sample. Some smaller regions of iodine segregation are also detected at the crack tip using NanoSIMS that do not appear in the chemical maps from STEM-EDX, this is probably due to the greater sensitivity of this technique to low concentration elements. However, it should be pointed that even with this greater sensitivity, iodine has not been detected at the crack tip regions of samples tested in iodised ethanol using NanoSIMS.

The results shown here provide the first direct evidence of iodine at a stress corrosion crack tip in irradiated $\mathrm{Zr}$, which provides unique insight into crack propagation mechanisms in reactor. The exact mechanism on a molecular scale of how I-SCC progresses has never been clear, with the surface mobility mechanism [27], (by which iodine forms bonds with zirconium atoms at the very crack tip surface until a volatile form is reached and material is removed) considered most likely [28-30]. It has been shown that bulk diffusion of iodine in zirconium is highly unlikely and so the zirconium-iodine reaction will occur at the open crack tip [31]. Direct evidence of this mechanism is lacking, however this result shows a clear correlation between iodine segregation and $\mathrm{Zr}$ material removal. The iodine segregation is also correlated to the oxygen distribution, suggesting that the open void left behind after the volatile zirconium iodide production has oxidised after the test. It should also be noted that the surface of the open crack tip is also decorated with iodine, suggesting iodine segregation was driving crack propagation and supporting the surface mobility mechanism. Unfortunately, it was not possible to obtain TKD orientation maps of the crack tip showing the iodine segregation due to a large volume fraction of FIB-induced hydrides in this sample. It is therefore not possible to assess the nature of the iodine segregation, i.e. whether it is 
as grain boundary or not. From the oxygen distribution, it is clear that this region contains a fine crack. lodine, supplied by the open crack tip, has formed a series of stable compounds, most likely these are the ones detected here, eventually forming a volatile phase that has removed $\mathrm{Zr}$ atoms preferentially. It is interesting to note that there is a significant crack adjacent to the iodine segregation that is not correlated to iodine segregation. One possible explanation is that this is showing direct competition between IG and TG cracking at the crack tip, whereby iodine is attacking the GB and the stress is causing localised deformation in the adjacent grain without the direct effect of iodine. It should also be noted that the other crack tip lifted out from the mandrel sample (figure $3 a$ ) did not show any significant iodine segregation and is dominated by TG cracking, indicating that TG cracking may not be directly caused by iodine segregation at the open crack tip.
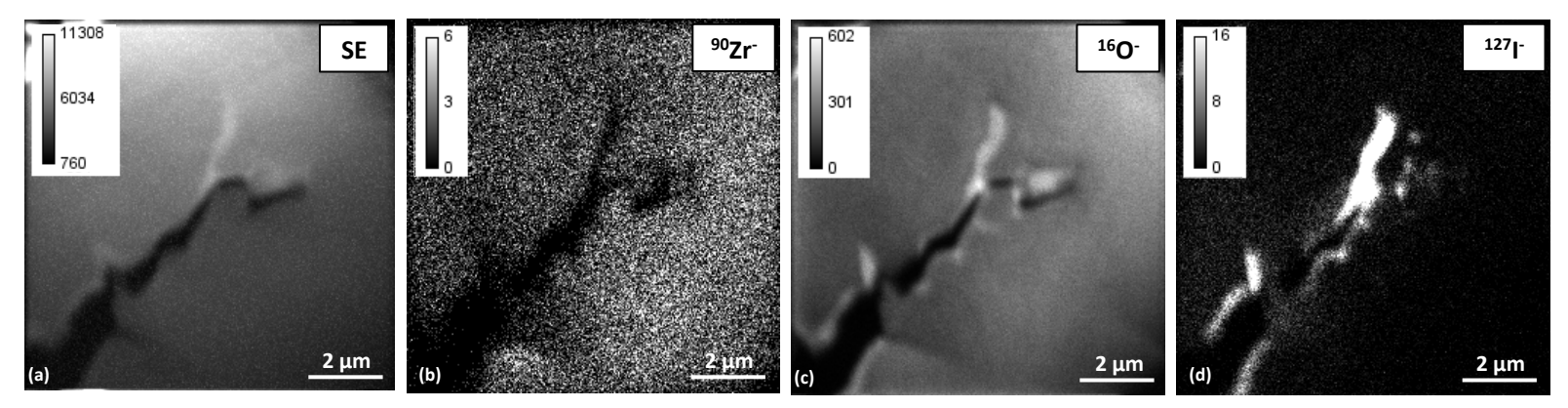

Figure 5: NanoSIMS maps of crack tip region from mandrel tested irradiated Zircaloy-2. Approximate region of analysis is highlighted in Figure 4 (a)

There are a large number of differences between the two experiments presented. The different loading regimes, fixed load or fixed strain rate, have been shown to control to alter the proportions of IG and TG failure modes, but have no effect on the orientation dependence[18,24]. Although the alloy composition is different, resulting in a different strength of the materials, alloy composition has not been observed to have a significant effect on I-SCC mechanics [32]. It is therefore thought the most likely cause of the different behaviours observed in this study, although only on a small number of grains, are the effects of irradiation and the corrosive media used. As discussed, previous literature suggests a strong preference for transgranular basal cleavage in non-irradiated material $[7,8,10-12]$. In contrast, the results shown here, albeit from a limited number of grains, indicate the occurrence of transgranular cracking on non-basal planes in neutron irradiated material. Previous work has shown that the effect of texture is less significant in neutron irradiated material [14], however this is the first time this phenomenon has been directly observed. The exact mechanism by which irradiation affects the crack process is not currently well 
understood, and experimental data is lacking [33]. It has previously been observed that irradiation can decrease $K_{I-S C C}$, the stress intensity factor at which I-SCC can occur [34,35], which suggests more options for TG crack progression becoming available following irradiation. Irradiation has a pronounced effect on deformation mechanisms in zirconium, altering the critical resolved sheer stress of slip systems, in particular on prismatic planes [36], and inducing strain localisation along dislocation channels [37], increasing susceptibility to I-SCC [38]. It is therefore possible that irradiation increases slip activity on prismatic and pyramidal planes allowing for fluting between cleavage planes that would have been possible in non-irradiated material. In addition, strain localisation due to irradiationinduced dislocation channelling and the resulting loss of ductility may increase I-SCC susceptibility further. This will be the subject of a more detailed study into I-SCC crack propagation in irradiated material that is currently in preparation.

\section{Conclusions}

The results presented here indicate a preference for non-basal transgranular cracking in the mandrel tested neutron irradiated material. Although there are a number of differences between the two experiments, such as alloy composition and loading geometry, the differences in crack morphology and orientation dependence are most likely due to both irradiation and the corrosive environment. The extensive grain boundary degradation present in the C-ring sample indicates that alcoholic iodine solutions are considerably more aggressive than gaseous iodine, which will have a dramatic effect on crack propagation mechanisms. The volatility of zirconium iodide, which is most likely different in the two environments, may also contribute to these observations. The results have also indicated that irradiation increases slip activity on prismatic and pyramidal planes allowing for fluting between cleavage planes that would have been possible in non-irradiated material. In addition, strain localisation due to irradiation-induced dislocation channelling and the resulting loss of ductility may increase I-SCC susceptibility further. The techniques developed here will allow for a more comprehensive study of crack tips formed on different alloys under different conditions in order to confirm these observations.

\section{Acknowledgements}

This work was funded by the EPSRC - PACIFIC [EP/L018616/1] Programme and is supported by EPSRC Centre for Doctoral Training in Nuclear Fission- Next Generation Nuclear [EP/L015390/1]. It has been carried out as part of the PACE consortium on Pellet Cladding 
Interaction, and the SCIP III program. The authors would like to thank Westinghouse Electric Sweden for providing the cladding materials used in this study. Christopher Jones and Katie L. Moore are acknowledged for NanoSIMS operation.

\section{Data Availability}

The raw and processed data required to reproduce these findings cannot be shared at this time as the data also forms part of an ongoing study.

\section{References}

[1] G.A. Ferrier, M. Farahani, J. Metzler, P.K. Chan, E.C. Corcoran, Mitigating the Stress Corrosion Cracking of Zircaloy-4 Fuel Sheathing: Siloxane Coatings Revisited, J. Nucl. Eng. Radiat. Sci. 2 (2016) 021004. doi:10.1115/1.4031620.

[2] S. Beguin, PCI-related constraints on EDF PWRs and associated challenges, in: Pellet-Clad Interact. Water React. Fuels, Organisation for Economic Co-Operation and Development Nuclear Energy Agency, 75 - Paris (France); 548 p; ISBN 92-64-01157-9; Worldcat; Jul 2005; p. 53-62; Seminar: Pellet-clad Interaction in Water Reactor Fuels; Aix-en-Provence (France); 9-11 Mar 2004;, 2005: pp. 53-62. doi.org/10.1787/9789264011588-en.

[3] S. Lozano-Perez, T. Yamada, T. Terachi, M. Schröder, C.A. English, G.D.W. Smith, C.R.M. Grovenor, B.L. Eyre, Multi-scale characterization of stress corrosion cracking of coldworked stainless steels and the influence of $\mathrm{Cr}$ content, Acta Mater. 57 (2009) 53615381. doi:10.1016/j.actamat.2009.07.040.

[4] S. Lozano-Perez, P. Rodrigo, L.C. Gontard, Three-dimensional characterization of stress corrosion cracks, J. Nucl. Mater. 408 (2011) 289-295. doi:10.1016/j.jnucmat.2010.11.068.

[5] L.N. Brewer, M.A. Othon, L.M. Young, T.M. Angeliu, Misorientation Mapping for Visualization of Plastic Deformation via Electron Back-Scattered Diffraction, (2018). doi:10.1017/S1431927606060120.

[6] M. Meisnar, M. Moody, S. Lozano-Perez, Atom probe tomography of stress corrosion crack tips in SUS316 stainless steels, Corros. Sci. 98 (2015) 661-671. doi:10.1016/j.corsci.2015.06.008.

[7] M.H.A. Piro, D. Sunderland, S. Livingstone, J. Sercombe, W. Revie, A. Quastel, K. Terrani, C. Judge, A Review of Pellet-Clad Interaction Behavior in Zirconium Alloy Fuel Cladding, in: Ref. Modul. Mater. Sci. Mater. Eng., Elsevier, 2017. doi:10.1016/B978-0-12-8035818.09799-X.

[8] B. Cox, Pellet-clad interaction ( $\mathrm{PCl}$ ) failures of zirconium alloy fuel cladding-a review, J. Nucl. Mater. 172 (1990) 249-292. https://doi.org/10.1016/0022-3115(90)90282-R.

[9] C. Gillen, A. Garner, A. Plowman, C.P. Race, T. Lowe, C. Jones, K.L. Moore, P. Frankel, Advanced 3D characterisation of iodine induced stress corrosion cracks in zirconium alloys, Mater. Charact. 141 (2018). doi:10.1016/j.matchar.2018.04.034.

[10] M. Peehs, H. Stehle, E. Steinberg, Out-of-Pile Testing of lodine Stress Corrosion Cracking In Zircaloy Tubing in Relation to the Pellet Cladding Interaction Phenomenon, in: Zircon. Nucl. Ind. Fourth Int. Symp., Stratford-upon-Avon, 1979: pp. 244-260. doi:https://doi.org/10.1520/STP681-EB. 
[11] R. Haddad, A. Dorado, Grain-by-grain study of the mechanisms of crack propagation during iodine stress corrosion cracking of Zircaloy-4, Zircon. Nucl. Ind. Tenth .... (1994). http://inis.iaea.org/search/search.aspx?orig_q=RN:26062624 (accessed October 20, 2014).

[12] D.B. Knorr, R.M. Pelloux, Effects of texture and microstructure on the propagation of iodine stress corrosion cracks in zircaloy, Metall. Trans. A. 13 (1982) 73-83. doi:10.1007/BF02642417.

[13] D.B. Knorr, R.M. Pelloux, L.F.P. Van Swam, Effects of material condition on the iodine SCC susceptibility of zircaloy-2 cladding, J. Nucl. Mater. 110 (1982) 230-245. doi:10.1016/0022-3115(82)90151-9.

[14] R.B. Adamson, Effect of texture on stress corrosion cracking of irradiated zircaloy in iodine, J. Nucl. Mater. 92 (1980) 363-365. doi:10.1016/0022-3115(80)90126-9.

[15] F. Onimus, I. Monnet, J.. Béchade, C. Prioul, P. Pilvin, A statistical TEM investigation of dislocation channeling mechanism in neutron irradiated zirconium alloys, J. Nucl. Mater. 328 (2004) 165-179. doi:10.1016/J.JNUCMAT.2004.04.337.

[16] L. Fournier, A. Serres, Q. Auzoux, D. Leboulch, G.S.S. Was, Proton irradiation effect on microstructure, strain localization and iodine-induced stress corrosion cracking in Zircaloy4, J. Nucl. Mater. 384 (2009) 38-47. doi:10.1016/j.jnucmat.2008.10.001.

[17] C. Anghel, A.-M.A. Holston, G. Lysell, R. Jakobsson, S. Karlsson, J. Flygare, E. Sund, Mahmood, T. Sheikh, An Out-of-Pile Method to Investigate lodine-induced SCC of Irradiated Cladding, Top Fuel 2009, Paris, Fr. Sept. 6-10, 2009. (2009) 823-834.

[18] C. Gillen, A. Garner, P. Frankel, Investigating lodine-Induced Stress Corrosion Cracking Of Zirconium Alloys Using Quantitative Fractography, Unpublished. (n.d.).

[19] J. Foster et al., ZIRLO Material Composition and Fabrication Processing, European Patent Specification EP 0475159 B1, 1996.

[20] A. International, ASTM B351 / B351M-13(2018), Standard Specification for Hot-Rolled and Cold-Finished Zirconium and Zirconium Alloy Bars, Rod, and Wire for Nuclear Application, 2018. doi:10.1520/B0351_B0351M-13R18.

[21] S. Lozano-Perez, A guide on FIB preparation of samples containing stress corrosion crack tips for TEM and atom-probe analysis, Micron. 39 (2008) 320-328. doi:10.1016/J.MICRON.2007.12.003.

[22] S.M. Hanlon, S.Y. Persaud, F. Long, A. Korinek, M.R. Daymond, A solution to FIB induced artefact hydrides in $\mathrm{Zr}$ alloys, J. Nucl. Mater. 515 (2019) 122-134. doi:10.1016/j.jnucmat.2018.12.020.

[23] A. Garner, A. Gholinia, P. Frankel, M. Gass, I. MacLaren, M. Preuss, The microstructure and microtexture of zirconium oxide films studied by transmission electron backscatter diffraction and automated crystal orientation mapping with transmission electron microscopy, Acta Mater. 80 (2014) 159-171. doi:10.1016/J.ACTAMAT.2014.07.062.

[24] S.Y. Park, J.H. Kim, M.H. Lee, Y.H. Jeong, Stress-corrosion crack initiation and propagation behavior of Zircaloy-4 cladding under an iodine environment, J. Nucl. Mater. 372 (2008) 293-303. doi:10.1016/j.jnucmat.2007.03.258.

[25] P. Jacques, F. Lefebvre, C. Lemaignan, Deformation-corrosion interactions for Zr alloys during I-SCC crack initiation: Part I: Chemical contributions, J. Nucl. Mater. 264 (1999) 239-248. doi:10.1016/S0022-3115(98)00501-7.

[26] S.B. Farina, G.S. Duffó, J.R. Galvele, Stress Corrosion Cracking of Zirconium and Zircaloy-4 in lodine-Alcoholic Solutions, Corrosion. 59 (2003) 436-442. doi:10.5006/1.3277575.

[27] J. Galvele, A stress corrosion cracking mechanism based on surface mobility, Corros. Sci. 27 (1987) 1-33. doi:10.1016/0010-938X(87)90117-X. 
[28] P.. Sidky, lodine stress corrosion cracking of Zircaloy reactor cladding: iodine chemistry (a review), J. Nucl. Mater. 256 (1998) 1-17. doi:10.1016/S0022-3115(98)00044-0.

[29] S.B. Farina, G.S. Duffo, J.R. Galvele, Stress corrosion cracking of zirconium and Zircaloy-4 in halide aqueous solutions, Corros. Sci. 45 (2003) 2497-2512. doi:10.1016/S0010938X(03)00075-1.

[30] M.L. Rossi, C.D. Taylor, First-principles insights into the nature of zirconium-iodine interactions and the initiation of iodine-induced stress-corrosion cracking, J. Nucl. Mater. 458 (2015) 1-10. doi:10.1016/j.jnucmat.2014.11.114.

[31] M.L. Rossi, C.D. Taylor, First-principles insights into the nature of zirconium-iodine interactions and the initiation of iodine-induced stress-corrosion cracking, J. Nucl. Mater. (2015). doi:10.1016/j.jnucmat.2014.11.114.

[32] R.L. Jones, D. Cubicciotti, B.C. Syrett, Effects of test temperature, alloy composition, and heat treatment on iodine-induced stress corrosion cracking of unirradiated zircaloy tubing, J. Nucl. Mater. 91 (1980) 277-292. doi:10.1016/0022-3115(80)90227-5.

[33] S.A. Nikulin, A.B. Rozhnov, Corrosion Cracking of Zirconium Cladding Tubes. A Review. 2. Effect of External Factors, Structure, and Properties of the Alloys, Met. Sci. Heat Treat. 47 (2005) 427-433. doi:10.1007/s11041-006-0006-1.

[34] Y.K. Bibilashvily, Y.N. Dolgov, B.I. Nesterov, V.V. Novikov, Propagation of stress corrosion cracks in Zr-1\% Nb claddings, J. Nucl. Mater. 224 (1995) 307-310. doi:10.1016/00223115(95)00114-X.

[35] Y.. Bibilashvili, A.. Medvedev, B.. Nesterov, V.. Novikov, V.. Golovanov, S.. Eremin, A.. Yurtchenko, Influence of irradiation on KISCC of Zr-1\%Nb claddings, J. Nucl. Mater. 280 (2000) 106-110. doi:10.1016/S0022-3115(00)00012-X.

[36] B. Bourdiliau, F. Onimus, C. Cappelaere, V. Pivetaud, P. Bouffioux, V. Chabretou, A. Miquet, Impact of Irradiation Damage Recovery During Transportation on the Subsequent Room Temperature Tensile Behavior of Irradiated Zirconium Alloys, in: Zircon. Nucl. Ind. 16th Int. Symp., ASTM International, 100 Barr Harbor Drive, PO Box C700, West Conshohocken, PA 19428-2959, 2012: pp. 929-953. doi:10.1520/STP152920120037.

[37] T.O.O. Erinosho, F.P.E.P.E. Dunne, Strain Localization and Failure in Irradiated Zircaloy with Crystal Plasticity, Int. J. Plast. 71 (2015) 170-194. doi:10.1016/J.IJPLAS.2015.05.008.

[38] D. Lee, R. Adamson, Modeling of Localized Deformation in Neutron Irradiated Zircaloy-2, in: Zircon. Nucl. Ind., ASTM International, 100 Barr Harbor Drive, PO Box C700, West Conshohocken, PA 19428-2959, n.d.: pp. 385-385-17. doi:10.1520/STP35582S. 